\title{
Comparative Effectiveness of Surgery for Traumatic Acute Subdural Hematoma in an Aging Population
}

\author{
Thomas A. van Essen, ${ }^{1,2,{ }^{*}}$ Mark D. Dijkman,, ${ }^{1, *}$ Maryse C. Cnossen, ${ }^{3}$ Walid Moudrous, ${ }^{4,5}$ \\ Hilko Ardon, ${ }^{6}$ Guus G. Schoonman, ${ }^{4}$ Ewout W. Steyerberg, ${ }^{3,7}$ Wilco C. Peul, ${ }^{1,2}$ \\ Hester F. Lingsma, ${ }^{3}$ and Godard C.W. de Ruiter ${ }^{2}$
}

\begin{abstract}
There is uncertainty as to the optimal initial management of patients with traumatic acute subdural hematoma, leading to regional variation in surgical policy. This can be exploited to compare the effect of various management strategies and determine best practices. This article reports such a comparative effectiveness analysis of a retrospective observational cohort of traumatic acute subdural hematoma patients in two geographically distinct neurosurgical departments chosen for their $-a$ priori defined - diverging treatment preferences. Region A favored a strategy focused on surgical hematoma evacuation, whereas region B employed a more conservative approach, performing primary surgery less often. Region was used as a proxy for preferred treatment strategy to compare outcomes between groups, adjusted for potential confounders using multivariable logistic regression with imputation of missing data. In total, 190 patients were included: 108 from region A and 82 from region B. There were 104 males (54.7\%). Matching current epidemiological developments, the median age was relatively high at 68 years (interquartile range [IQR], 54-76). Baseline characteristics were comparable between regions. Primary evacuation was performed in $84 \%$ of patients in region A and in $65 \%$ of patients in region B $(p<0.01)$. Mortality was lower in region A (37\% vs. $45 \%, p=0.29)$, as was unfavorable outcome (53\% vs. $62 \%, p=0.23)$. The strategy favoring surgical evacuation was associated with significantly lower odds of mortality (odds ratio [OR]: $0.43 ; 95 \%$ confidence interval [CI]: 0.21-0.88) and unfavorable outcome (OR: 0.53; 95\% CI: 0.27-1.02) 3-9 months post-injury. Therefore, in the aging population of patients with acute subdural hematoma, a treatment strategy favoring emergency hematoma evacuation might be associated with lower odds of mortality and unfavorable outcome.
\end{abstract}

Keywords: acute subdural hematoma; epidemiology; surgery; traumatic brain injury

\section{Introduction}

I 1983, GelPKe AND COLleagues compared survival after severe traumatic brain injury (TBI) between two neurosurgical departments in the Netherlands. They concluded that of the $18 \%$ difference in survival rate between centers, $10.5 \%$, was accounted for by differences in baseline characteristics, and that the remaining $7.5 \%$ could reflect differences in management strategies. ${ }^{1}$ This study can be regarded as comparative effectiveness research (CER) avant la lettre in the field of TBI. CER aims to relate naturally occurring treatment variation to differences in patient-relevant outcomes, measured in day-to-day clinical settings and broad populations. ${ }^{2}$ This is increasingly recognized as a powerful alternative to randomized controlled trials (RCTs). ${ }^{3}$ Many surgical trials have been hampered by protracted recruitment periods ${ }^{4,5}$ and financial constraints, ${ }^{6}$ while providing limited benefit to clinical management. ${ }^{5,7-9}$ Moreover, CER could be worthwhile because, partly as a result of current treatment guidelines featuring low class evidence, considerable between-center differences in the clinical management of TBI are seen. ${ }^{10-16}$

"To operate or not?" is a burning clinical question every surgeon frequently has to answer. In the case of traumatic acute subdural hematoma (ASDH), whether the patient will benefit from direct surgical evacuation of the hematoma remains a contested issue. Although prompt surgery has been associated with improved survival, ${ }^{17-20}$ reported mortality rates remain high, ranging from $40 \%$ to $60 \%$ in surgically treated patients of all trauma severities. ${ }^{21}$ Conversely, it has been suggested that certain patients with a poor

Departments of ${ }^{1}$ Neurosurgery and ${ }^{7}$ Medical Statistics and Bioinformatics, Leiden University Medical Center, Leiden, The Netherlands.

${ }^{2}$ Department of Neurosurgery, Medial Center Haaglanden, The Hague, The Netherlands.

${ }^{3}$ Center for Medical Decision Sciences, Department of Public Health, Erasmus Medical Center, Rotterdam, The Netherlands.

Departments of ${ }^{4}$ Neurology and ${ }^{6}$ Neurosurgery, Elisabeth-Tweesteden Hospital, Tilburg, The Netherlands.

${ }^{5}$ Department of Neurology, Maasstad Hospital, Rotterdam, The Netherlands.

*The first two authors contributed equally 
prognosis may not be treated at all, ${ }^{22,23}$ and patients with minimal symptomatology or comatose ASDH patient with a minimal hematoma without extracranial explanations for his/her coma can successfully be treated conservatively. ${ }^{24-27}$ Conservative treatment comprises observation, serial neurological examinations, and, sometimes, placement of an intracranial pressure (ICP) monitor with non-operative/medical management of intracranial hypertension when appropriate.

Therefore, the decision to operate or not poses a clinical challenge for the neurosurgeon, and should preferably be aided by guidelines based on comparison of treatment regimes. However, RCTs have never been conducted, and comparative observational cohort studies with balanced treatment groups are unavailable. The most widely used surgical guidelines published by the Brain Trauma Foundation are consequently made up of weak evidence. ${ }^{21}$ Therefore, clinical decision making is presently influenced by neurosurgeons' individual preferences for, or familiarity with, treatment of traumatic ASDH. As a result, management strategies vary considerably among neurosurgical departments, even among those within dedicated level 1 trauma centers with protocolled emergency medicine schemes. ${ }^{10-12}$ Clearly, there is a need for comparative studies to elucidate the optimal treatment of traumatic ASDH. As randomized surgical trials are not practically feasible because of the moral boundaries of treating physicians, the comparison of cohorts among homogenous regions managing ASDH in different ways may be the best available alternative to assess the effectiveness of surgical therapy.

The objective of this study was to compare mortality and functional outcome between an immediate surgical and a conservative, less surgery-driven approach for the treatment of traumatic ASDH.

\section{Methods}

This study was conducted and reported according to the criteria of the Strengthening the Reporting of Observational Based Studies (STROBE) statement. ${ }^{28}$

\section{The Dutch healthcare system and regional treatment variation}

Healthcare in the Netherlands is uniformly accessible to all patients, with equal distribution of resources among hospitals. Neurosurgical care for patients with TBI is provided at 11 level 1 trauma centers, serving separate areas according to regional referral policies. Training and licensing are equal among these centers. However, neurosurgical practices differ across these centers, probably because of local surgical customs handed down over the years: a recent survey assessing whether neurosurgeons would perform an operation on various patients with ASDH showed considerable between-center variation among Dutch neurosurgeons' attitudes toward patients with traumatic ASDH. ${ }^{12}$ Based on this questionnaire, the centers that showed the most divergent view on whether to operate acutely or not in cases reflecting the whole clinical TBI severity spectrum $(90.0 \%$ vs. $14.3 \%$ for moderate TBI, $90.0 \%$ vs. $42.9 \%$ for severe TBI) were chosen before the start of the current study. In both centers, the same Brain Trauma Foundation guideline-based intensive care unit (ICU) protocol for (refractory) raised ICP is employed whereas the neurosurgical departments do not employ a surgical protocol (e.g., the Brain Trauma Foundation guideline).

\section{Study design, setting and population}

Two patient cohorts were retrospectively identified at two neurosurgical departments, in geographically separate regions. Neurosurgeons from region A treat TBI patients at two neurosurgically collaborating hospitals within level 1 trauma centers and advocate primary surgical treatment of ASDH (through hematoma evacuation with or without decompression). Neurosurgeons from region $\mathrm{B}$ operate in a single level 1 trauma center and opt for surgery less often, suggesting a more conservative approach. ${ }^{12}$ Both regions serve a homogeneous population of $\sim 2,000,000$ people. Regions A and $\mathrm{B}$ employed 16 and 18 neurosurgeons, respectively.

\section{Eligibility criteria}

The Dutch registry system for hospital funding appoints diagnosis and treatment codes to all patients visiting a hospital. Using this system as a screening tool, we consecutively identified all patients treated by a neurosurgeon for TBI between 2008 and 2012 in both regions. Thereafter, the national trauma registry was checked for any missed inclusions. ${ }^{29}$ A broad set of inclusion criteria was applied: patients were included if they had sustained brain injury with direct presentation to the emergency room, were $>16$ years of age, and showed a hyperdense, crescent shaped lesion - indicative of an ASDH - on the computed tomography (CT) scan. Exclusion criteria were penetrating injury, non-traumatic ASDH, ASDH secondary to an earlier procedure, and presenting with concomitant intracranial focal lesions (i.e., intracerebral hematoma or epidural hematoma) that required emergency surgery. Patients withheld from treatment because of severe comorbidity or because they were deemed unsalvageable were also excluded on the premises that the outcome would have been the same regardless of treatment.

\section{Variables}

Data from electronic patient files were gathered on demographics, medical history, use of anticoagulants or antiplatelet agents, injury-related variables, radiological variables, treatment variables, complications, and outcome variables.

Injury-related variables included trauma mechanism, first emergency room Glasgow Coma Scale (GCS) score, focal neurological symptoms (paresis, aphasia, or cranial nerve deficit), pupillary light reflex, clinical deterioration (i.e., a decrease of $>1$ point on the GCS, new abnormal pupillary light reflex, or new focal neurological symptoms, from the time of first assessment), presence of significant extracranial injury, and primary presentation to the study hospitals.

Radiological variables were assessed from the first CT scan. They included clot thickness, midline shift, patency of the basal cisterns, presence of cranial fractures, and presence of concomitant intracranial hemorrhage (subarachnoid hemorrhage, epidural hematoma, contralateral subdural hematoma, intraventricular hemorrhage, or intracerebral hematoma/contusion). We also noted if a second preoperative CT scan was made, and whether it showed radiological deterioration (i.e., presence of new focal lesion or $>5 \mathrm{~mm}$ increase in hematoma thickness).

Therapy-related variables included type of management (conservative or surgical), type of surgery (craniotomy, decompressive craniectomy [DC], or other), use of an ICP monitor, and any delayed surgical procedures performed after the initial treatment was received. The maximum diameter of the DC was measured from postoperative CT scans. Patients were considered surgically treated when a report was made on the indication and the surgical procedure was started after the last CT performed in the emergency room. Patients were considered conservatively managed when the neurosurgeon on call said not to operate after the CT. Patients who were primarily conservatively managed could require surgery later on, after secondary deterioration.

All complications requiring medical attention during admission (e.g., antibiotic treatment for infection) were noted. Complications were defined as intracranial (e.g., seizure, hydrocephalus), cardiovascular (e.g., arrhythmia, ischemia), respiratory (e.g., respiratory insufficiency, hypoxia), metabolic (e.g., electrolyte disturbances, renal failure), infections (e.g., pneumonia, wound infection), or other. 
Outcome measures were mortality at discharge and functional outcome according to the Glasgow Outcome Score (GOS). Functional outcome was judged from outpatient follow-up letters at 3-9 months post-trauma, and dichotomized into favorable (GOS 4-5) or unfavorable (GOS 1-3) categories. ${ }^{30}$

Two authors from region A collected all data. To ensure uniform and unbiased collection, both contributors independently gathered data according to a standardized collection sheet. In case of uncertainty, variables were coded after consensus was reached through discussion.

This study was approved by the ethics committees of the two participating hospitals.

\section{Statistical analysis}

The median and interquartile range (IQR) were calculated for continuous variables, and frequencies were reported along with percentages for categorical variables. To test for differences in patient characteristics, treatments, and outcomes between regions, we used Pearson's $\chi^{2}$ test for categorical, and the Mann-Whitney $U$ test for continuous variables. In order to provide a summary baseline prognostic score for both regions, a multivariable logistic regression model, based on all available variables featured in the Corticosteroid Randomization After Significant Head injury (CRASH)-CT brain injury prognosis model (age, GCS score, pupil reactivity to light, major extracranial injury, midline shift $>5 \mathrm{~mm}$, traumatic subarachnoid hemorrhage, and obliteration of the basal cisterns), was used to calculate predicted probabilities for mortality and unfavorable outcome. ${ }^{31}$

To assess the effect of treatment strategy on outcome, we used region as a proxy for treatment strategy. That strategy correlates with more surgeries (and possibly other aspects; see the Discussion section). Assuming that regional variation was the only determinant of treatment strategy, the region in which patients were treated was used as grouping variable to compare outcomes. A multivariable regression model was used to estimate the effect of treatment strategy on outcome, adjusted for the confounders age, GCS score, pupil reactivity, and hematoma thickness. In addition, subgroup analysis restricted to patients with mild to moderate TBI (GCS 915) was performed. The regression analyses were performed using a multiple imputed data set created with the automatic imputation method of the impute function of SPSS. Missing data were reported for all variables at baseline. Results were considered statistically significant if $p<0.05$. All analyses were performed using IBM's SPSS version 23 .

\section{Results}

\section{Participants and presentation}

A total of 612 cases were screened $(n$, region $\mathrm{A}=294 ; n$, region $\mathrm{B}=318$ ) of which 195 cases met the eligibility criteria: 109 from region $A$ and 86 from region $B$. Five cases were excluded because of severe comorbidity ( $n$, region $\mathrm{A}=1)$, or were deemed unsalvageable ( $n$, region $\mathrm{B}=4$ ) (Fig. 1).

The study population included 104 males $(54.7 \%)$ and the median age was 68 years (IQR, 54-76). Regions did not differ significantly on age, sex, use of anticoagulants, injury mechanism, GCS score, occurrence of focal neurological symptoms, pupillary examination, or frequency of major extracranial injury (Table 1). Region A received more primary referrals than region B (63\% vs. $21 \%, p<0.01)$. Patients in region B more often experienced clinical deterioration ( $34 \%$ vs. $51 \%, p<0.01$ ). Region A had larger median hematoma thickness $(14 \mathrm{~mm}$ vs. $10 \mathrm{~mm}, p<.01)$, larger median midline shift $(12 \mathrm{~mm}$ vs. $9 \mathrm{~mm}, p<0.01)$, and fewer cranial fractures $(28 \%$ vs. $44 \%, p<0.03)$. The baseline CRASH-CT-predicted risk of unfavorable outcome was similar: $56 \%$ in region A and $53 \%$ in region $\mathrm{B}(p=0.18)$. Missing values per variable used in both univariable and multivariable regression analyses did not exceed $5.8 \%$ (Table 1).

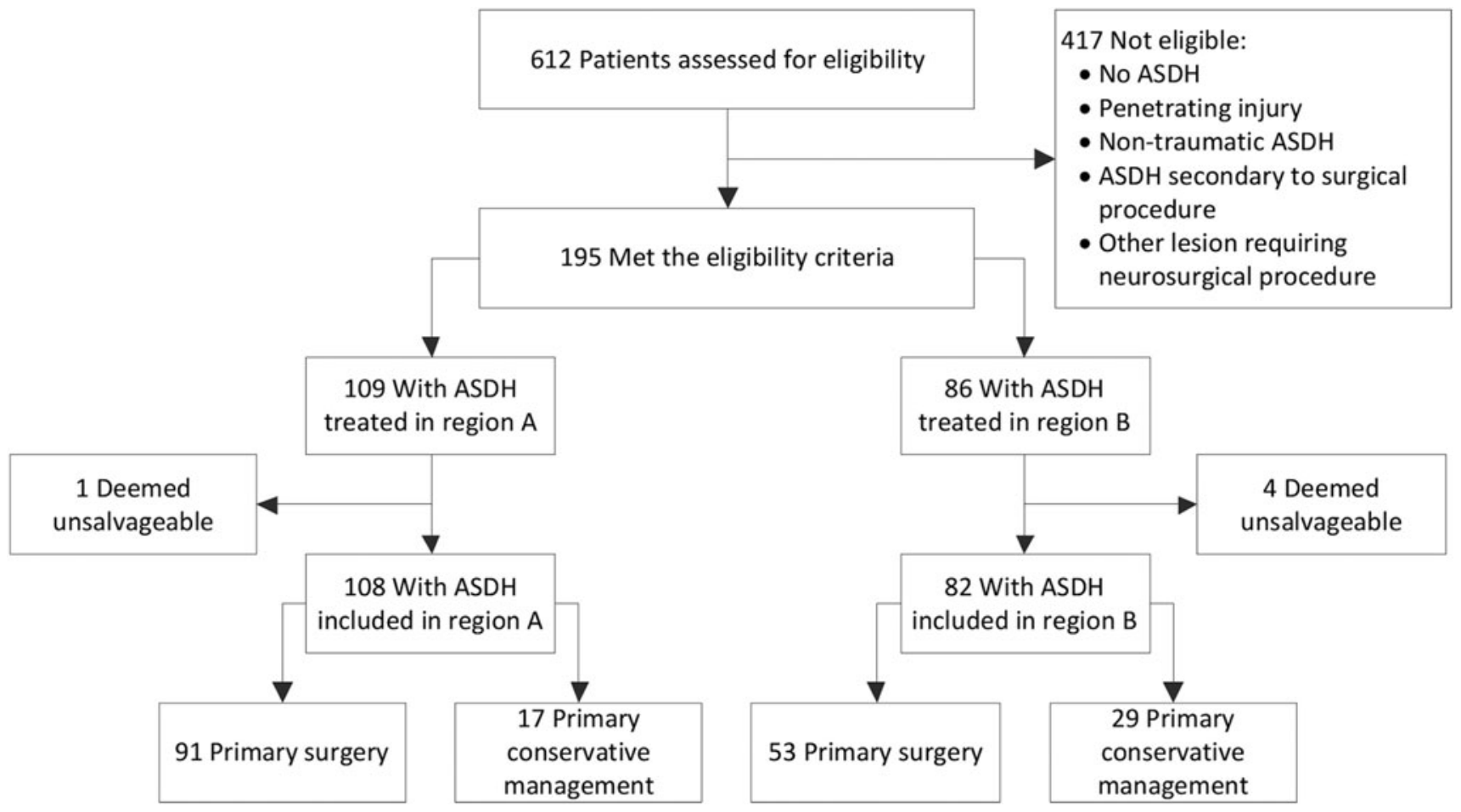

FIG. 1. Patient inclusion flow chart showing the number of patients included in each region (region A: liberal surgical policy, region B: more conservative policy) and the definitive treatment received (either primary surgical or conservative). 
Table 1. Clinical and Radiological Variables for the Entire Study Population

\begin{tabular}{|c|c|c|c|}
\hline Variable, $\%$ missing & Region A $(\mathrm{n}=108)$ & Region $B(\mathrm{n}=82)$ & $\mathrm{p}$ value \\
\hline Age, median (IQR), years, $0 \%$ & $70(54-78)$ & $65(53-74)$ & 0.07 \\
\hline Male sex, no. (\%), $0 \%$ & $57 / 108(53 \%)$ & $47 / 82(57 \%)$ & 0.56 \\
\hline History of diabetes, no. (\%), $1.6 \%$ & $14 / 105(13 \%)$ & $10 / 82(12 \%)$ & 1.00 \\
\hline Vascular history, no. (\%), $1.6 \%$ & $60 / 105(57 \%)$ & $45 / 82(55 \%)$ & 0.769 \\
\hline \multicolumn{4}{|l|}{ Anticoagulant, no. (\%), $1.6 \%$} \\
\hline Anticoagulants & $34 / 107(32 \%)$ & $18 / 80(23 \%)$ & 0.19 \\
\hline Platelet inhibitors & $20 / 107(20 \%)$ & $16 / 80(19 \%)$ & 1.00 \\
\hline \multicolumn{4}{|l|}{ Mechanism of injury, no. (\%), $2.5 \%$} \\
\hline Fall & $58 / 104(56 \%)$ & $45 / 81(56 \%)$ & 1.00 \\
\hline Assault & $5 / 104(5 \%)$ & $1 / 81(1 \%)$ & 0.23 \\
\hline Motor vehicle accident & $12 / 104(12 \%)$ & $13 / 81(16 \%)$ & 0.39 \\
\hline Fall from bicycle & $12 / 104(12 \%)$ & $11 / 81(14 \%)$ & 0.82 \\
\hline Other & $17 / 104(16 \%)$ & $11 / 81(14 \%)$ & 0.68 \\
\hline Primary presentation, no. $(\%), 0 \%$ & $67 / 106(63 \%)$ & $17 / 82(21 \%)$ & $<0.01$ \\
\hline Focal neurological symptoms, no. (\%), $24.7 \%$ & $42 / 79(53 \%)$ & $28 / 64(44 \%)$ & 0.31 \\
\hline \multicolumn{4}{|l|}{ Abnormal pupils, no. (\%), $5.3 \%$} \\
\hline One & $13 / 101(13 \%)$ & $12 / 79(15 \%)$ & 0.67 \\
\hline Two & $14 / 101(14 \%)$ & $9 / 79(12 \%)$ & 0.66 \\
\hline First GCS score, median (IQR), $2.6 \%$ & $9(6-14)$ & $12(7-15)$ & 0.48 \\
\hline Initial motor (M) score, median (IQR), $29.5 \%$ & $6(5-6)$ & $5(4-6)$ & 0.04 \\
\hline \multicolumn{4}{|l|}{ TBI severity, no. (\%), $1.6 \%$} \\
\hline Mild (GCS 13-15) & $40 / 107(37 \%)$ & $29 / 80(36 \%)$ & 1.00 \\
\hline Moderate (GCS 9-12) & $18 / 107(17 \%)$ & $20 / 80(25 \%)$ & 0.20 \\
\hline Severe (GCS 3-8) & $49 / 107(46 \%)$ & $31 / 80(39 \%)$ & 0.37 \\
\hline Clinical deterioration, no. $(\%), 0 \%$ & $37 / 108(34 \%)$ & $44 / 82(54 \%)$ & $<0.01$ \\
\hline GCS score after deterioration, median (IQR), 5.2\% & $8(4-13)$ & $8(5-13)$ & 0.45 \\
\hline Major extracranial injury, no. (\%), $3.2 \%$ & $12 / 102(12 \%)$ & $11 / 82(13 \%)$ & 0.82 \\
\hline \multicolumn{4}{|l|}{ CT characteristics } \\
\hline Thickness, median (IQR), mm, 5.8\% & $14(9-18)$ & $10(7-14)$ & $<0.01$ \\
\hline Midline shift, median (IQR), mm, $4.2 \%$ & $12(7-16)$ & $8(3-13)$ & $<0.01$ \\
\hline Basal cisterns obliterated, no. (\%), $4.2 \%$ & $39 / 101(39 \%)$ & $24 / 81(30 \%)$ & 0.22 \\
\hline Concomitant SAH, no. (\%), $2.1 \%$ & $24 / 104(23 \%)$ & $23 / 82(28 \%)$ & 0.50 \\
\hline Concomitant contusion, no. ( $\%), 2.6 \%$ & $20 / 104(19 \%)$ & $25 / 81(31 \%)$ & 0.08 \\
\hline Concomitant EDH, no. $(\%), 2.1 \%$ & $7 / 104(7 \%)$ & $4 / 82(5 \%)$ & 0.76 \\
\hline Concomitant SDH, no. (\%), $2.1 \%$ & $8 / 104(8 \%)$ & $11 / 82(13 \%)$ & 0.23 \\
\hline Concomitant IVH, no. (\%), $2.1 \%$ & $2 / 104(2 \%)$ & $2 / 82(2 \%)$ & 1.00 \\
\hline Cranial fracture, no. $(\%), 2.1 \%$ & $30 / 106(28 \%)$ & $35 / 80(44 \%)$ & 0.03 \\
\hline Second CT scan, no. $(\%), 1.6 \%$ & $23 / 105(22 \%)$ & $19 / 82(23 \%)$ & 0.86 \\
\hline Radiological deterioration, $2.4 \%$ & $14 / 22(64 \%)^{a}$ & $10 / 19(53 \%)^{\mathrm{a}}$ & 0.54 \\
\hline $\begin{array}{l}\text { Predicted unfavorable outcome based on CRASH-CT } \\
\text { variables (GOS } \leq 3, \%) \text {, median (IQR), } 12.6 \%\end{array}$ & $56 \%(42-75 \%)$ & $53 \%(32-70 \%)$ & 0.18 \\
\hline
\end{tabular}

${ }^{\text {a}}$ Percentage of all second CT scans.

IQR, interquartile range; GCS, Glasgow Coma Scale; TBI, traumatic brain injury; CT, computed tomography; SAH, subarachnoid hemorrhage; EDH, epidural hematoma; SDH, subdural hematoma; IVH, intraventricular hemorrhage; CRASH, Corticosteroid Randomization After Significant Head Injury; GOS, Glasgow Outcome Score.

\section{Interventions}

Patients in region A more often received emergency surgery $(84 \%$ vs. $65 \%, p<0.01)$ or an ICP sensor ( $38 \%$ vs. $15 \%, p<0.01)$. When undergoing surgery, patients were more likely to undergo $\mathrm{DC}$ in region $\mathrm{B}(32 \%$ vs. $55 \%, p<0.01)$. The median size of DC in region $\mathrm{B}$ was somewhat larger $(110 \mathrm{~mm}$ vs. $116 \mathrm{~mm}, p=0.15)$. Both regions had a similar rate of delayed procedures $(15 \%$ vs. $16 \%$, $p=0.57$ ) and complications (Table 2).

\section{The effect of treatment strategy}

Hospital mortality in region A was $37 \%$, compared with $45 \%$ in region $\mathrm{B}(p=0.29)$. Also, in region $\mathrm{A}, 53 \%$ had an unfavorable outcome after 3-9 months, compared with $62 \%$ in region $\mathrm{B}$ $(p=0.23)$. Region showed a favorable effect for a more aggressive treatment strategy (region A) in both unadjusted (mortality odds ratio $[\mathrm{OR}], 0.75 ; 95 \%$ confidence interval $[\mathrm{CI}], 0.42-1.10$; unfavorable outcome OR, 0.74; 95\% CI, 0.41-1.02) and adjusted analysis (mortality OR, 0.43; 95\% CI, 0.21-0.88; unfavorable outcome OR, 0.53; 95\% CI, 0.27-1.02). Subgroup analysis restricted to patients with mild to moderate TBI $(n=110)$ showed a similar effect for both mortality (adjusted OR, 0.34; 95\% CI, 0.130.91 ) and unfavorable outcome (adjusted OR, 0.33 ; 95\% CI 0.14 0.77) (Table 3). A post-hoc analysis with adjustment for DC and primary referral confirmed the main results (Tables S1 and S2) (see online supplementary material at http://www.liebertpub.com). 
Table 2. Hospital Course Variables for the Entire Study Population

\begin{tabular}{|c|c|c|c|}
\hline Variable, \% missing & Region A $(\mathrm{n}=108)$ & Region $B(\mathrm{n}=82)$ & $\mathrm{p}$ values \\
\hline ICP monitor placed, no. (\%), $1.1 \%$ & $40 / 106(38 \%)$ & $12 / 82(15 \%)$ & $<0.01$ \\
\hline \multicolumn{4}{|l|}{ Emergency surgery, $0 \%$} \\
\hline Total, no. $(\%)$ & $91 / 108(84 \%)$ & $53 / 82(65 \%)$ & $<0.01$ \\
\hline Craniotomy, no. (\%) & $60 / 91(66 \%)^{\mathrm{a}}$ & $24 / 53(45 \%)^{a}$ & 0.02 \\
\hline DC, no. $(\%)$ & $29 / 91(32 \%)^{\mathrm{a}}$ & $29 / 53(55 \%)^{\text {a }}$ & $<0.01$ \\
\hline Other, no. (\%) & $2 / 91(2 \%)^{a}$ & $0 / 53(0 \%)^{a}$ & 0.53 \\
\hline Size of DC, median (IQR) & $110(97-115)$ & $116(103.5-127)$ & 0.15 \\
\hline \multicolumn{4}{|l|}{ Delayed surgery, no. (\%), $2.6 \%$} \\
\hline Total & $16 / 103(15 \%)$ & $16 / 82(19 \%)$ & 0.57 \\
\hline Craniotomy & $4 / 16(25 \%)^{a}$ & $7 / 15(44 \%)^{\mathrm{a}}$ & 0.46 \\
\hline DC & $6 / 16(38 \%)^{a}$ & $4 / 15(25 \%)^{a}$ & 0.70 \\
\hline Burrhole & $4 / 16(25 \%)^{\mathrm{a}}$ & $3 / 15(19 \%)^{a}$ & 1.00 \\
\hline Other & $2 / 16(13 \%)^{\mathrm{a}}$ & $2 / 15(13 \%)^{\mathrm{a}}$ & 1.00 \\
\hline \multicolumn{4}{|l|}{ Complications, no. (\%), $7.4 \%$} \\
\hline Total & $56 / 95(59 \%)$ & $50 / 81(62 \%)$ & 0.76 \\
\hline Intracranial & $30 / 95(32 \%)$ & $29 / 81(36 \%)$ & 0.63 \\
\hline Cardiovascular & $13 / 95(14 \%)$ & $17 / 81(21 \%)$ & 0.23 \\
\hline Respiratory & $4 / 95(4 \%)$ & $9 / 81(11 \%)$ & 0.15 \\
\hline Metabolic & $3 / 95(3 \%)$ & $9 / 81(11 \%)$ & 0.07 \\
\hline Infection & $30 / 95(32 \%)$ & $18 / 81(22 \%)$ & 0.18 \\
\hline Other & $1 / 100(1 \%)$ & $7 / 81(9 \%)$ & 0.02 \\
\hline
\end{tabular}

${ }^{\text {a}}$ Percentage within group of emergency or delayed procedures

ICP, intracranial pressure; IQR, interquartile range; DC, decompressive craniectomy

\section{Effect of surgery on patient level}

In multivariable analysis, surgery was associated with higher odds of in-hospital mortality (OR, 6.6; 95\% CI, 1.43-30.8) and unfavorable outcome (OR, 1.56, 95\% CI: 0.64-3.82, Table 4).

\section{Discussion}

This study compared an aggressive surgical policy to a more conservative management strategy for traumatic ASDH. Although the study groups were comparable in terms of baseline prognosis,

Table 3. Output of Logistic Regression Analyses

\begin{tabular}{|c|c|c|}
\hline Analysis type & $\begin{array}{l}\text { Mortality } \\
\text { at discharge }\end{array}$ & $\begin{array}{l}\text { Unfavorable } \\
\text { outcome } \\
\text { at 3-9 months }\end{array}$ \\
\hline \multicolumn{3}{|l|}{ All patients } \\
\hline $\begin{array}{l}\text { Univariable }(n=190), \\
\text { OR }(95 \% \mathrm{CI})\end{array}$ & $0.75(0.42-1.10)$ & $0.74(0.41-1.02)$ \\
\hline $\begin{array}{l}\text { Multivariable }(n=190), \\
\text { OR }(95 \% \mathrm{CI})^{\mathrm{a}}\end{array}$ & $0.43(0.21-0.88)$ & $0.53(0.27-1.02)$ \\
\hline \multicolumn{3}{|l|}{$\begin{array}{l}\text { Subgroup of mild-moderate } \\
\text { TBI patients }\end{array}$} \\
\hline $\begin{array}{l}\text { Univariable }(n=110), \\
\text { OR }(95 \% \mathrm{CI})\end{array}$ & $0.51(0.22-1.18)$ & $0.45(0.21-0.96)$ \\
\hline $\begin{array}{l}\text { Multivariable }(n=110) \text {, } \\
\quad \text { OR }(95 \% \mathrm{CI})^{\mathrm{b}}\end{array}$ & $0.34(0.13-0.91)$ & $0.33(0.14-0.77)$ \\
\hline
\end{tabular}

Numbers are odds ratios and $95 \%$ confidence intervals for the effect of treatment strategy on mortality and unfavorable outcome (Glasgow Outcome Score $\leq 3$ ).

${ }^{a}$ Controlling for age, GCS score, pupillary reactivity, and hematoma thickness.

${ }^{\mathrm{b}}$ Controlling for age, GCS score, and hematoma thickness.

OR, odds ratio; CI, confidence interval; TBI, traumatic brain injury; GCS, Glasgow coma scale. patients were significantly more likely to undergo emergency surgery in the expected region. A primarily surgically focused strategy was associated with a lower in-hospital mortality and unfavorable outcome.

Estimating causal treatment effects in non-randomized data is often impossible because of confounding by indication. Some of the baseline imbalance can be corrected through stratification or multivariable modeling (among other selection or analysis techniques). However, when we compared a surgical with a non-operative treatment on patient level in this study (Table 4), in contrast to comparing treatment strategies on a center level, emergency surgery was associated with worse outcome even after adjusting for confounders. This suggests unmeasured confounding by indication, causing misleading effect estimates. The region-based comparison is a major strength of this study. It allowed for a "natural experiment" in which patients were "allocated" to one of both policies based on where the accident occurred, and not based on their baseline characteristics. This provided an opportunity for an analysis that likely has a considerable reduction of unmeasured

Table 4. Output of Logistic Regression Analyses

\begin{tabular}{llc}
\hline Analysis type & $\begin{array}{c}\text { Mortality } \\
\text { at discharge }\end{array}$ & $\begin{array}{c}\text { Unfavorable } \\
\text { outcome } \\
\text { at 3-9 months }\end{array}$ \\
\hline $\begin{array}{l}\text { Effect of surgery on patient } \\
\quad \text { level }\end{array}$ & & \\
Univariable, OR (95\% CI) & $9.7(3.3-28.6)$ & $3.5(1.7-7.3)$ \\
Multivariable, OR $(95 \% \mathrm{CI})^{\mathrm{a}}$ & $6.6(1.4-30.8)$ & $1.6(0.6-3.8)$ \\
\hline
\end{tabular}

Numbers are odds ratios and $95 \%$ confidence intervals for the effect of surgery on mortality and unfavorable outcome (Glasgow Outcome Score $\leq 3$ ).

${ }^{a}$ Controlling for age, Glasgow Coma Scale score, pupillary reactivity and hematoma thickness.

OR, odds ratio; $\mathrm{CI}$, confidence interval 
confounding compared with an analysis comparing the actual treatment received. Based on the balance between groups with regard to measured confounders, we may speculate that there is also balance in unmeasured confounders. This assumption, however, cannot be statistically proven (as would neither be possible nor rational in an $\mathrm{RCT}$ ).

A weakness is that we compared surgical strategy in only two centers. It cannot be excluded that differences between regions in other aspects of care; for example, other treatments or organization of care, caused the difference in outcome in addition to the surgical approach. To account for these differences in such a hospital-level approach, more centers are generally needed to disentangle the care aspects that correlate with the treatment decision of interest. Therefore, we need to interpret our results with caution. The wide $\mathrm{CI}$ of the effect estimates clearly underwrites this statement even more. A study with more hospitals is needed to allow adjustment for differences between the hospitals other than in the treatment of interest.

An obvious limitation in this context, although inherent to the observational nature, is that the region-based approach did not completely eliminate all differences in baseline confounders. Patients from region A presented directly to the study hospital more often $(63 \%$ vs. $21 \%, p<0.01)$. Primary presentation to a neurosurgical center improves patient outcome and has a close relationship with time to surgery, ${ }^{32}$ both of which may have contributed to the lower rates of mortality and unfavorable outcome observed in region $\mathrm{A}$. The higher incidence of clinical deterioration in region $\mathrm{B}$ may be consequent to the larger number of secondary referrals. On the other hand, region B is characterized by relatively smaller hematoma thickness and midline shift (on the same time point CT), while having a higher proportion of accompanying contusions. However, the possible resulting confounding is adjusted for appropriately. Moreover, apparently, the imbalances are evenly spread out because the cohorts of both regions have a similar prognosis in the validated CRASH model. Therefore, they were similar for the most important predictors of outcome on arrival to the study hospital.

Another limitation may be that the data collectors could not be blinded for hospital of admission, which might give rise to information bias. To minimize potential bias, a standardized and controlled data collection protocol was followed. Nevertheless, we need to consider that the GOS was estimated from clinic discharge and visit letters, instead of a structured interview.

Further, this study needs an important note on generalizability. This is a relatively elderly population, with a high proportion of fall-related ASDH (56\%). Recent comparable series of patients with ASDH of varying severities report mean ages of 55-58 years. ${ }^{19,33,34}$ The advanced age in this study should come as no surprise considering the aging population in developed countries, but should be kept in mind when applying its results. Also, for a clinician to determine to which patients these results are relevant she/he needs to consider for what kind of ASDH patients neurosurgery is consulted in the Netherlands. An unknown number of mild cases reviewed by the neurologist or neurology resident without consultation of a neurosurgeon were not included. This explains the relatively small number of conservatively treated patients (24\% overall) in our cohort compared with recent studies, reporting $74-83 \%$ of ASDHs being treated nonoperatively. ${ }^{33,34}$ And, although much less common, this also applies to very severe cases, not operated on because of an extremely poor prognosis. Because the mild cases constitute the majority of TBIs, and this patient category undoubtedly has an above-average prognosis, applying the favorable results of surgery to all ASDH patients would not be reasonable. Although both the age and consultation patterns might influence the generalizability, we nonetheless consider the current selection useful in research on surgical decision making, because our cohort is representative of the ASDH population currently presented to neurosurgeons.

\section{Role of surgery for ASDH}

Our results are in line with other recent studies reporting a positive effect of early/aggressive surgical management, mainly for severe TBI, on mortality and functional outcome. ${ }^{15,35} \mathrm{~A}$ recent report showed that this could also be true for routine use of invasive ICP measurement for severe TBI. ${ }^{14}$ On the other hand, when restricting to surgery, and the complete patient domain; that is, ASDH patients with mild to severe TBI, previous studies have reported worse or similar outcomes in patients undergoing surgical treatment. ${ }^{24-26,32,36,37}$ However, all studies compared outcomes between surgically and non-operatively treated patients at the patient level rather than at a regional level, potentially leading to confounding by indication, because more severely injured patients are more likely to be operated on. As mentioned, when performing a conventional analysis on our cohort, surgery was associated with increased mortality and unfavorable outcome in our data. Hence, the distorting effect of confounding by indication in TBI supports the use of a comparative effectiveness approach when an RCT is not feasible for whatever reason.

When discussing the role of surgery in TBI, the use of DC cannot be left unmentioned. Region B used DC significantly more often, despite equal distribution of prognostic factors. This could reflect another variation in surgical strategy or be consequent to the higher number of secondary referrals, resulting in presentation at a later stage, when brain swelling has started to occur. There is limited evidence on the use of primary DC in the treatment of ASDH, and studies comparing craniotomy with DC are likely confounded by indication as well. ${ }^{38-40}$ Consequently, the effect of more frequent use of DC in region B on our outcome measures is uncertain. The planned Randomised Evaluation of Surgery with Craniectomy for Patients Undergoing Evacuation for ASDH (RESCUE-ASDH) trial will use an experimental comparative effectiveness design to clarify the value of DC as a primary treatment in severe TBI. ${ }^{11}$

This study reports an example of the practice variation in surgical treatment of traumatic ASDH. Advantage can be taken of this variation for CER. Larger cohorts with more hospitals are required to perform robust analyses to explore the ability of this method to infer causality, and with enough statistical power to study specific substrata according to age and trauma severity. The availability of such cohorts, with prospectively gathered, trial- quality data from real-world settings, will allow initiatives such as Neuro-traumatology Quality Registry (Net-QuRe) and Collaborative European NeuroTrauma Effectiveness Research in Traumatic Brain Injury (CENTER-TBI) to answer urgent clinical questions to provide the much needed guidance for the treatment of TBI. ${ }^{41}$

\section{Conclusion}

In this comparative study of surgery for ASDH, an aggressive management strategy favoring emergency evacuation of the hematoma was associated with better outcome. This conclusion derives from a contemporary cohort of relatively elderly traumatic ASDH patients that reflects the current population presented to a neurosurgeon. Larger, prospective, comparative studies with more 
hospitals are needed to confirm this effect of surgery and to explore generalizability.

\section{Acknowledgments}

This study was funded by the European Union Seventh Framework Program (grant 602150) for CENTER-TBI and Hersenstichting Nederland (Dutch Brain Foundation, project PS2014-06) for the Neurotraumatology Quality Registry (Net-QuRe). The authors have no industry affiliation.

Portions of this work were presented orally as a plenary lecture at the 12th Symposium of the International Neurotrauma Society, Capetown, South Africa, in 2016.

\section{Author Disclosure Statement}

No competing financial interests exist.

\section{References}

1. Gelpke, G.J., Braakman, R., Habbema, J.D., and Hilden, J. (1983). Comparison of outcome in two series of patients with severe head injuries. J. Neurosurg. 59, 745-750.

2. Timmons, S.D., and Toms, S.A. (2012). Comparative effectiveness research in neurotrauma. Neurosurg. Focus 33, E3.

3. Maas, A.I., Menon, D.K., Lingsma, H.F., Pineda, J.A., Sandel, M.E., and Manley, G.T. (2012). Re-orientation of clinical research in traumatic brain injury: report of an international workshop on comparative effectiveness research. J. Neurotrauma 29, 32-46.

4. Hutchinson, P.J., Corteen, E., Czosnyka, M., Mendelow, A.D., Menon, D.K., Mitchell, P., Murray, G., Pickard, J.D., Rickels, E., Sahuquillo, J., Servadei, F., Teasdale, G.M., Timofeev, I., Unterberg, A., and Kirkpatrick, P.J. (2006). Decompressive craniectomy in traumatic brain injury: the randomized multicenter RESCUEicp study (www.RESCUEicp.com). Acta Neurochir. Suppl. 96, 17-20.

5. Chesnut, R.M., Temkin, N., Carney, N., Dikmen, S., Rondina, C., Videtta, W., Petroni, G., Lujan, S., Pridgeon, J., Barber, J., Machamer, J., Chaddock, K., Celix, J.M., Cherner, M., Hendrix, T., and Global Neurotrauma Research, G. (2012). A trial of intracranial-pressure monitoring in traumatic brain injury. N. Engl. J. Med. 367, 2471-2481.

6. Gregson, B.A., Rowan, E.N., Francis, R., McNamee, P., Boyers, D., Mitchell, P., McColl, E., Chambers, I.R., Unterberg, A., and Mendelow, A.D. (2015). Surgical Trial In Traumatic intraCerebral Haemorrhage (STITCH): a randomised controlled trial of early surgery compared with initial conservative treatment. Health Technol. Assess. $19,1-138$.

7. Cooper, D.J., Rosenfeld, J. V, Murray, L., Arabi, Y.M., Davies, A.R., D’Urso, P., Kossmann, T., Ponsford, J., Seppelt, I., Reilly, P., Wolfe, R., Investigators, D.T., Australian, and New Zealand Intensive Care Society Clinical Trials, G. (2011). Decompressive craniectomy in diffuse traumatic brain injury. N Engl J Med 364, 1493-1502.

8. Maas, A.I., Roozenbeek, B., and Manley, G.T. (2010). Clinical trials in traumatic brain injury: past experience and current developments. Neurotherapeutics 7, 115-126.

9. Tolias, C.M., and Bullock, M.R. (2004). Critical appraisal of neuroprotection trials in head injury: what have we learned? NeuroRx 1, 71-79.

10. Kolias, A.G., Belli, A., Li, L.M., Timofeev, I., Corteen, E.A., Santarius, T., Menon, D.K., Pickard, J.D., Kirkpatrick, P.J., and Hutchinson, P.J. (2012). Primary decompressive craniectomy for acute subdural haematomas: results of an international survey. Acta Neurochir. 154, 1563-1565.

11. Kolias, A.G., Scotton, W.J., Belli, A., King, A.T., Brennan, P.M., Bulters, D.O., Eljamel, M.S., Wilson, M.H., Papadopoulos, M.C., Mendelow, A.D., Menon, D.K., Hutchinson, P.J., Network, U.K.N.R., and group, R.-A. collaborative. (2013). Surgical management of acute subdural haematomas: current practice patterns in the United Kingdom and the Republic of Ireland. Br. J. Neurosurg. 27, 330-333.

12. van Essen, T.A., de Ruiter, G.C., Kho, K.H., and Peul, W.C. (2017). Neurosurgical treatment variation of traumatic brain injury: evaluation of acute subdural hematoma management in Belgium and The Netherlands. J. Neurotrauma 34, 881-889.
13. Dawes, A.J., Sacks, G.D., Cryer, H.G., Gruen, J.P., Preston, C., Gorospe, D., Cohen, M., McArthur, D.L., Russell, M.M., MaggardGibbons, M., Ko, C.Y., and Los Angeles County Trauma, C. (2015). Compliance with evidence-based guidelines and interhospital variation in mortality for patients with severe traumatic brain injury. JAMA Surg. 150, 965-972.

14. Alali, A.S., Fowler, R.A., Mainprize, T.G., Scales, D.C., Kiss, A., de Mestral, C., Ray, J.G., and Nathens, A.B. (2013). Intracranial pressure monitoring in severe traumatic brain injury: results from the American College of Surgeons Trauma Quality Improvement Program. J. Neurotrauma $30,1737-1746$.

15. Hartings, J.A., Vidgeon, S., Strong, A.J., Zacko, C., Vagal, A., Andaluz, N., Ridder, T., Stanger, R., Fabricius, M., Mathern, B., Pahl, C., Tolias, C.M., Bullock, M.R., and Co-Operative Studies on Brain Injury, D. (2014). Surgical management of traumatic brain injury: a comparative-effectiveness study of 2 centers. J. Neurosurg. 120, 434446.

16. Compagnone, C., Murray, G.D., Teasdale, G.M., Maas, A.I., Esposito, D., Princi, P., D'Avella, D., Servadei, F., and European Brain Injury Consortium (2005). The management of patients with intradural posttraumatic mass lesions: a multicenter survey of current approaches to surgical management in 729 patients coordinated by the European Brain Injury Consortium. Neurosurgery 57, 1183-1192.

17. Seelig, J.M., Becker, D.P., Miller, J.D., Greenberg, R.P., Ward, J.D., and Choi, S.C. (1981). Traumatic acute subdural hematoma: major mortality reduction in comatose patients treated within four hours. N. Engl. J. Med. 304, 1511-1518.

18. Wilberger Jr., J.E., Harris, M., and Diamond, D.L. (1991). Acute subdural hematoma: morbidity, mortality, and operative timing. J. Neurosurg. 74, 212-218.

19. Leitgeb, J., Mauritz, W., Brazinova, A., Janciak, I., Majdan, M., Wilbacher, I., and Rusnak, M. (2012). Outcome after severe brain trauma due to acute subdural hematoma. J. Neurosurg. 117, 324333.

20. Matsushima, K., Inaba, K., Siboni, S., Skiada, D., Strumwasser, A.M., Magee, G.A., Sung, G.Y., Benjaminm, E.R., Lam, L., and Demetriades, D. (2015). Emergent operation for isolated severe traumatic brain injury: Does time matter? J. Trauma Acute Care Surg. 79, 838842.

21. Bullock, M.R., Chesnut, R., Ghajar, J., Gordon, D., Hartl, R., Newell, D.W., Servadei, F., Walters, B.C., and Wilberger, J.E. (2006). Surgical management of acute subdural hematomas. Neurosurgery 58, S16-S24.

22. Scotter, J., Hendrickson, S., Marcus, H.J., and Wilson, M.H. (2015). Prognosis of patients with bilateral fixed dilated pupils secondary to traumatic extradural or subdural haematoma who undergo surgery: a systematic review and meta-analysis. Emerg. Med. J. 32, 654-659.

23. Nijboer, J.M.M., van der Naalt, J., and ten Duis, H.J. (2010). Patients beyond salvation?. Various categories of trauma patients with a minimal Glasgow Coma Score. Injury 41, 52-57.

24. Croce, M.A., Dent, D.L., Menke, P.G., Robertson, J.T., Hinson, M.S., Young, B.H., Donovan, T.B., Pritchard, F.E., Minard, G., Kudsk, K.A., et al. (1994). Acute subdural hematoma: nonsurgical management of selected patients. J. Trauma 36, 820-827.

25. Servadei, F., Nasi, M.T., Cremonini, A.M., Giuliani, G., Cenni, P., and Nanni, A. (1998). Importance of a reliable admission Glasgow Coma Scale score for determining the need for evacuation of posttraumatic subdural hematomas: a prospective study of 65 patients. J. Trauma 44 , 868-873.

26. Feliciano, C.E., and De Jesus, O. (2008). Conservative management outcomes of traumatic acute subdural hematomas. P. R. Health Sci. J. $27,220-223$.

27. Mathew, P., Oluoch-Olunya, D.L., Condon, B.R., and Bullock, R. (1993). Acute subdural haematoma in the conscious patient: outcome with initial non-operative management. Acta Neurochir. 121, 100108.

28. von Elm, E., Altman, D.G., Egger, M., Pocock, S.J., Gotzsche, P.C., Vandenbroucke, J.P., and STROBE Initiative. (2014). The Strengthening the Reporting of Observational Studies in Epidemiology (STROBE) Statement: guidelines for reporting observational studies. Lancet 370, 1453-1457.

29. Goslings, J.C., and Gouma, D.J. (2009). Trauma registration. A promising, but difficult process [in Dutch]. Ned. Tijdschr. Geneeskd. $153,744-746$. 
30. Jennett, B., and Bond, M. (1975). Assessment of outcome after severe brain damage. Lancet 1, 480-484.

31. Collaborators, M.C.T., Perel, P., Arango, M., Clayton, T., Edwards, P., Komolafe, E., Poccock, S., Roberts, I., Shakur, H., Steyerberg, E., and Yutthakasemsunt, S. (2008). Predicting outcome after traumatic brain injury: practical prognostic models based on large cohort of international patients. BMJ 336, 425-429.

32. Stone, J.L., Lowe, R.J., Jonasson, O., Baker, R.J., Barrett, J., Oldershaw, J.B., Crowell, R.M., and Stein, R.J. (1986). Acute subdural hematoma: direct admission to a trauma center yields improved results. J. Trauma 26, 445-450.

33. Bajsarowicz, P., Prakash, I., Lamoureux, J., Saluja, R.S., Feyz, M., Maleki, M., and Marcoux, J. (2015). Nonsurgical acute traumatic subdural hematoma: what is the risk? J. Neurosurg. 123, 1-8.

34. Ryan, C.G., Thompson, R.E., Temkin, N.R., Crane, P.K., Ellenbogen, R.G., and Elmore, J.G. (2012). Acute traumatic subdural hematoma: current mortality and functional outcomes in adult patients at a Level I trauma center. J. Trauma Acute Care Surg. 73, 1348-1354.

35. Hedges, J.R., Newgard, C.D., Veum-Stone, J., Selden, N.R., Adams, A.L., Diggs, B.S., Arthur, M., and Mullins, R.J. (2009). Early neurosurgical procedures enhance survival in blunt head injury: propensity score analysis. J. Emerg. Med. 37, 115-123.

36. Dent, D.L., Croce, M.A., Menke, P.G., Young, B.H., Hinson, M.S., Kudsk, K.A., Minard, G., Pritchard, F.E., Robertson, J.T., and Fabian, T.C. (1995). Prognostic factors after acute subdural hematoma. J. Trauma 39, 33-36.

37. Tierney, K.J., Nayak, N. V, Prestigiacomo, C.J., and Sifri, Z.C. (2015). Neurosurgical intervention in patients with mild traumatic brain injury and its effect on neurological outcomes. J. Neurosurg. $124,538-545$.
38. Li, L.M., Kolias, A.G., Guilfoyle, M.R., Timofeev, I., Corteen, E.A., Pickard, J.D., Menon, D.K., Kirkpatrick, P.J., and Hutchinson, P.J. (2012). Outcome following evacuation of acute subdural haematomas: a comparison of craniotomy with decompressive craniectomy. Acta Neurochir. 154, 1555-1561.

39. Chen, S.H., Chen, Y., Fang, W.K., Huang, D.W., Huang, K.C., and Tseng, S.H. (2011). Comparison of craniotomy and decompressive craniectomy in severely head-injured patients with acute subdural hematoma. J. Trauma 71, 1632-1636.

40. Woertgen, C., Rothoerl, R.D., Schebesch, K.M., and Albert, R. (2006). Comparison of craniotomy and craniectomy in patients with acute subdural haematoma. J. Clin. Neurosci. 13, 718-721.

41. Maas, A.I., Menon, D.K., Steyerberg, E.W., Citerio, G., Lecky, F., Manley, G.T., Hill, S., Legrand, V., Sorgner, A., Participants, C.-T., and Investigators. (2015). Collaborative European NeuroTrauma Effectiveness Research in Traumatic Brain Injury (CENTER-TBI): a prospective longitudinal observational study. Neurosurgery 76, $67-80$.

Address correspondence to:

Thomas A. van Essen, MD, MSc

Department of Neurosurgery

Leiden University Medical Center

Albinusdreef 2

2333 ZA, Leiden, Mailing Zone J-11R

The Netherlands

E-mail: t.a.van_essen@lumc.nl 